
\title{
28 Research Suare \\ Relationship Between Hyperhidrosis and Hypothalamic Injury in Patients With Mild Traumatic Brain Injury
}

Sung Ho Jang

Yeungnam University

Hyeok Gyu Kwon ( $\boldsymbol{\nabla}$ khg0715@hanmail.net )

Eulji University

\section{Research Article}

Keywords: Diffusion tensor imaging, Hypothalamus, Hyperhidrosis, Paroxysmal sympathetic hyperactivity, Mild traumatic brain injury.

Posted Date: December 3rd, 2020

DOI: https://doi.org/10.21203/rs.3.rs-112407/v1

License: (c) (i) This work is licensed under a Creative Commons Attribution 4.0 International License.

Read Full License 


\section{Abstract}

Hyperhidrosis is clinical symptom of various diseases and is an important clinical feature of paroxysmal sympathetic hyperactivity(PSH). Traumatic brain injury(TBI) is the most common condition associated with PSH, and PSH has been mainly reported in moderate and severe TBI. However, very little has been reported on PSH or hyperhidrosis in mild TBI patients. In this study, we used diffusion tensor imaging(DTI) to investigate the relationship between hyperhidrosis and hypothalamic injury in patients with mild TBI. Seven patients with hyperhidrosis after mild TBI and 21 healthy controls were recruited for this study. The Hyperhidrosis Disease Severity Scale was used for evaluation of sweating at the time of DTI scanning. The fractional anisotropy(FA) and apparent diffusion coefficient(ADC) DTI parameters were measured in the hypothalamus. In the patient group, the FA values for both sides of the hypothalamus were significantly lower than those of the control group $(p<0.05)$. By contrast, the ADC values for both sides of the hypothalamus were significantly higher in the patient group than in the control group $(p<0.05)$. In conclusion, we detected hypothalamic injuries in patients who showed hyperhidrosis after mild TBI. Based on the results, it appears that hyperhidrosis in patients with mild TBI is related to hypothalamic injury.

\section{Introduction}

Hyperhidrosis is a condition characterized by abnormal excessive sweating, that is, sweating in excess of that required for the regulation of body temperature ${ }^{1}$. It worsens quality of life from psychological, emotional, and social perspectives ${ }^{2}$. In addition, hyperhidrosis is an important clinical feature of paroxysmal sympathetic hyperactivity (PSH), a syndrome that causes episodes of increased sympathetic nervous system activity in patients with an acquired brain injury ${ }^{3,4}$. While PSH can arise from many types of the acquired brain injury, traumatic brain injury (TBI) is presented to be the most common disease associated with $\mathrm{PSH}$, and $\mathrm{PSH}$ has been mainly reported in patients with moderate and severe $\mathrm{TBI}^{4-8}$. However, very little is known about PSH or hyperhidrosis in patients with mild $\mathrm{TBI}^{9}$.

The central regulatory center for thermoregulatory sweating is located in the hypothalamus ${ }^{10,11}$, and hypothalamic injury has been suggested as an important pathophysiological mechanism associated with hyperhidrosis in patients with brain injuries ${ }^{12-18}$. Exact evaluation of the hypothalamus in the live human brain has been limited because of anatomical features: very small size and deep location within the white matter ${ }^{19,20}$. Nonetheless, diffusion tensor imaging (DTI) allows measurement of the hypothalamus by using various DTI parameters in the live human brain and several studies have reported on the relationship between hypothalamic injury and various clinical features, including narcolepsy, depression, and cognitive fatigue, exhibited in various brain diseases including TBI, hypoxic-ischemic brain injury, and multiple sclerosis ${ }^{21-25}$. However, no study on the relationship between hyperhidrosis and hypothalamic injury has been reported. 
In this study, by using DTI, we investigated the relationship between hyperhidrosis and hypothalamic injury in patients with mild TBI.

\section{Methods}

\section{Subjects}

Seven patients (male: 2 , female: 5 , mean age: $51.4 \pm 6.7$ years, range: $39-60$ years) with mild TBI and 21 age- and sex-matched healthy control subjects (male: 7, female: 14 , mean age: $48.1 \pm 9.8$ years, range: 35-60 years) with no previous history of neurological, physical, or psychiatric illness were recruited for this study (Table 1). The following inclusion criteria were used in the recruitment of patients: (1) loss of consciousness (LOC) for $<30$ minutes, post-traumatic amnesia (PTA) for $\leq 24$ hours, and initial Glasgow Coma Scale (GCS) score of 13-1526; (2) no specific lesion observed on brain MRI (T1-weighted, T2weighted, and fluid-attenuated inversion recovery images); (3) more than one month elapsed since onset of TBI; (4) presence of hyperhidrosis after the onset of head trauma; and (5) no history of previous head trauma and neurologic or psychiatric disease. Because this study was conducted retrospectively, we were not able to obtain patient's consent. However, we can obtain the healthy subject's consent. As a result, all healthy subjects understood the purpose of this study and provided written, informed consent prior to participation. In addition, our study protocol excluding patient's consent and including healthy subject's consent was approved (YUMC-2019-06-032) by the Institutional Review Board of a Yeungnam University Hospital. The study was carried out in accordance with relevant guidelines and regulations.

\section{Clinical evaluation}

The previously developed Hyperhidrosis Disease Severity Scale (HDSS) was used to evaluate the subjects' sweating characteristics at the time of DTI scanning. The HDSS score ranged from 1 to 4 with score $=1$ signifying that sweating is never noticeable and never interferes with daily activities, score 2 indicating that sweating is tolerable but sometimes interferes with daily activities, score 3 indicating that sweating is barely tolerable and frequently interferes with daily activities; and score 4 signifying that sweating is intolerable and always interferes with daily activities ${ }^{27}$. The average HDSS score in the patient group was $2.4 \pm 0.8$ (Table 1). Clinical features of PSH present in each patient and the HDSS scores for each patient are presented in Table 2.

\section{Diffusion tensor imaging}

A multi-channel head coil on a 1.5 T Philips Gyroscan Intera (Philips, Ltd, Best, Netherlands) with 32 gradients was used for acquisition of DTI data. DTI imaging parameters were set as follows: acquisition matrix $=96 \times 96$; reconstructed to matrix $=192 \times 192$; field of view $=240 \mathrm{~mm} \times 240 \mathrm{~mm}$; repetition time $=$ $10,398 \mathrm{~ms}$; echo time $=72 \mathrm{~ms}$; parallel imaging reduction factor $=2$; echo-planar imaging factor $=59 ; b=$ $1000 \mathrm{~s} / \mathrm{mm}^{2}$; number of excitations $=1$; and slice thickness $=2.5 \mathrm{~mm}$. Eddy current-induced image distortions were removed using affine multi-scale two-dimensional registration as provided by the Oxford Centre for Functional Magnetic Resonance Imaging of Brain Software Library ${ }^{28}$. DTI-Studio software 
(CMRM, Johns Hopkins Medical Institute, Baltimore, MD, USA) was used for evaluation of the hypothalamus, which was identified by establishing the optic tract as the anterior boundary and the mammillary body as the posterior boundary at the level of the upper midbrain (Fig. 1) 21,29 . The fractional anisotropy (FA) and apparent diffusion coefficient (ADC) values were measured in the area identified as the hypothalamus ${ }^{30}$.

\section{Statistical analysis}

Statistical analyses were performed using SPSS software (v. 25.0; SPSS, Chicago, IL, USA). An independent $t$-test was used to determine the significance of differences in the FA and ADC values of the hypothalamus between the patient and control groups. The significance level for the obtained $p$-value was set at 0.05 .

\section{Results}

A summary of the DTI parameter results for the hypothalamus in the patient and control groups is shown in Table 3. In the patient group, the FA values for both sides of the hypothalamus were significantly lower than those of the control group $(p<0.05)$. By contrast, the ADC values for both sides of the hypothalamus were significantly higher in the patient group than in the control group $(p<0.05)$.

\section{Discussion}

In the current study, by using DTI hypothalamic injuries were investigated in patients exhibiting hyperhidrosis following mild TBI. Our results are summarized as follows: 1 ) the patient group had lower FA values and higher ADC values for both sides of the hypothalamus than those of the control group ( $p<$ 0.05 ); and 2) all patients exhibited a HDSS score of two or greater. The FA and ADC values are commonly used to demonstrate the status of brain structures in patients with brain injuries. The FA value indicates the degree of directionality of water diffusion; consequently, it reflects fiber density, axonal diameter, and white matter myelination, whereas the ADC value indicates the magnitude of water diffusion ${ }^{31}$. Therefore, a low FA value combined with a high ADC value is indicative of brain injury ${ }^{32}$. In the present study, the FA and $A D C$ values indicate the presence of hypothalamic injuries in the patient group and these injuries might be related to the concurrent presence of hyperhidrosis in the patient group.

Hyperhidrosis is a clinical feature of $\mathrm{PSH}^{3,4}$, and $\mathrm{PSH}$ presence is determined, in the absence of other potential causes such as uncontrolled sepsis or airway obstruction, by the transient presence of four of the following six criteria: fever, tachycardia (heart rate $>120$ beats/min or $>100$ beats/min if treated with a beta-blocker), hypertension (systolic blood pressure $>160 \mathrm{mmHg}$ or pulse pressure $>80 \mathrm{mmHg}$ ), tachypnea (respiratory rate $>30$ breaths/min), hyperhidrosis, and extensor posturing or severe dystonia 3,4. Although the pathophysiological mechanisms of PSH have not clearly elucidated, two main mechanisms have been suggested: 1) simple disconnection of cortical inhibitory centers such as the insula and cingulate cortex to the brain areas responsible for supraspinal control of sympathetic tone 
(hypothalamus, diencephalon, and brainstem); and 2) the excitatory:inhibitory ratio model in which paroxysms are driven by abnormal processing of afferent stimuli within the spinal cord following disconnection of descending inhibitory pathways s, $^{7,12,33}$. Although no patient in this study satisfied the diagnostic criteria for PSH, we suggest that the hyperhidrosis exhibited by our patients may be considered a clinical feature indicative of PSH because the hypothalamus is an important area for the development of $\mathrm{PSH}^{7,12,33}$. We assume that the reason our patients could not meet the diagnostic criteria of PSH is related to their head injuries being milder than that of moderate and severe TBI.

The hypothalamus has been regarded as a key regulator of thermoregulatory sweating ${ }^{10,11}$. Several studies have reported associations between hypothalamic injury and hyperhidrosis in patients with brain injury, including those with cerebral infarct and multiple sclerosis ${ }^{15-18}$. Therefore, the results of our study appear to be consistent with those of the abovementioned studies ${ }^{15-18}$. To the best of our knowledge, this is the first study to demonstrate an association between hyperhidrosis and hypothalamic injury in patients with mild TBI. However, limitations of this study should be considered. First, the technique for measurement of DTI parameters is operator-dependent, particularly during the definition of the region of interest; regardless, we attempted to define consistently the hypothalamus by using definite boundaries in this study ${ }^{34}$. Second, a small number of subjects were recruited in this study. Therefore, conduct of further prospective studies that enroll larger numbers of subjects should be encouraged.

In conclusion, by using DTI, we detected hypothalamic injury in patients who showed hyperhidrosis after mild TBI. Based on the results, it appears that hyperhidrosis exhibited by patients with mild TBI can be related to hypothalamic injury. Our results suggest that DTI could be useful in detecting hypothalamic injury, injuries that may not be detected on conventional brain MRI in patients with mild TBI.

\section{Declarations}

\section{Acknowledgements}

This work was supported by the Medical Research Center Program (2015R1A5A2009124) through the National Research Foundation of Korea (NRF) funded by the Ministry of Science, ICT and Future Planning.

\section{Competing interests}

The author(s) declare no competing interests.

\section{Author contributions}

S.H.J: Conceiving and designing the study, manuscript writing, data acquisition. H.G.K.: Manuscript development, data analysis, manuscript writing and manuscript authorization.

\section{Data availability}


The dataset used and/or analyzed during current study are available from the corresponding author on reasonable request.

\section{References}

1. James, W. D., Berger, T. G., Elston, D. M. \& Odom, R. B. Andrews' diseases of the skin : clinical dermatology. 10th edn, (Saunders Elsevier, 2006).

2. Vary, J. C., Jr. Selected Disorders of Skin Appendages-Acne, Alopecia, Hyperhidrosis. Med Clin North Am. 99, 1195-1211 (2015).

3. Rabinstein, A. A. Paroxysmal sympathetic hyperactivity in the neurological intensive care unit. Neurol Res. 29, 680-682 (2007).

4. Perkes, I. E., Menon, D. K., Nott, M. T. \& Baguley, I. J. Paroxysmal sympathetic hyperactivity after acquired brain injury: a review of diagnostic criteria. Brain Inj. 25, 925-932 (2011).

5. Perkes, I., Baguley, I. J., Nott, M. T. \& Menon, D. K. A review of paroxysmal sympathetic hyperactivity after acquired brain injury. Ann Neurol. 68, 126-135 (2010).

6. Hasen, M., Almojuela, A. \& Zeiler, F. A. Autonomic Dysfunction and Associations with Functional and Neurophysiological Outcome in Moderate/Severe Traumatic Brain Injury: A Scoping Review. J Neurotrauma. 36, 1491-1504 (2019).

7. Meyfroidt, G., Baguley, I. J. \& Menon, D. K. Paroxysmal sympathetic hyperactivity: the storm after acute brain injury. Lancet Neurol. 16, 721-729 (2017).

8. Mathew, M. J., Deepika, A., Shukla, D., Devi, B. I. \& Ramesh, V. J. Paroxysmal sympathetic hyperactivity in severe traumatic brain injury. Acta Neurochir (Wien). 158, 2047-2052 (2016).

9. Jang, S. H., Kwon, Y. H. \& Lee, S. J. Tachycardia in a patient with mild traumatic brain injury. Clin Auton Res. (2019).

10. Schlereth, T., Dieterich, M. \& Birklein, F. Hyperhidrosis--causes and treatment of enhanced sweating. Dtsch Arztebl Int. 106, 32-37 (2009).

11. Low, P. A. \& Benarroch, E. E. Clinical autonomic disorders. 3rd edn, (Lippincott Williams \& Wilkins, 2008).

12. Baguley, I. J., Heriseanu, R. E., Cameron, I. D., Nott, M. T. \& Slewa-Younan, S. A critical review of the pathophysiology of dysautonomia following traumatic brain injury. Neurocrit Care. 8, 293-300 (2008).

13. Pranzatelli, M. R., Pavlakis, S. G., Gould, R. J. \& De Vivo, D. C. Hypothalamic-midbrain dysregulation syndrome: hypertension, hyperthermia, hyperventilation, and decerebration. J Child Neurol. 6, 115122 (1991).

14. Thorley, R. R., Wertsch, J. J. \& Klingbeil, G. E. Acute hypothalamic instability in traumatic brain injury: a case report. Arch Phys Med Rehabil. 82, 246-249 (2001).

15. Diamond, A., Kenney, C., Almaguer, M. \& Jankovic, J. Hyperhidrosis due to deep brain stimulation in a patient with essential tremor. Case report. J Neurosurg. 107, 1036-1038 (2007). 
16. Smith, C. D. A hypothalamic stroke producing recurrent hemihyperhidrosis. Neurology. 56, 1394-1396 (2001).

17. Sakashita, Y., Kakuta, K., Kakuma, K. \& Matsuda, H. Unilateral persistent hyperhidrosis after ischemic stroke. Rinsho Shinkeigaku. 32, 454-456 (1992).

18. Ueno, M., Tokunaga, Y., Terachi, S., Gondo, K. \& Hara, T. Asymmetric sweating in a child with multiple sclerosis. Pediatr Neurol. 23, 74-76 (2000).

19. Afifi, A. K. \& Bergman, R. A. Functional neuroanatomy : text and atlas. 2nd edn, (Lange Medical Books/McGraw-Hill, 2005).

20. Baroncini, M. et al. MRI atlas of the human hypothalamus. Neuroimage. 59, 168-180 (2012).

21. Jang, S. H. \& Kwon, H. G. Injury of the Hypothalamus in Patients With Hypoxic-Ischemic Brain Injury: A Diffusion Tensor Imaging Study. Am J Phys Med Rehabil. 97, 160-163 (2018).

22. Menzler, K. et al. DTI reveals hypothalamic and brainstem white matter lesions in patients with idiopathic narcolepsy. Sleep Med. 13, 736-742 (2012).

23. Shen, Y. et al. Depressive symptoms in multiple sclerosis from an in vivo study with TBSS. Biomed Res Int. 2014, 148465 (2014).

24. Jang, S. H., Yi, J. H., Kim, S. H. \& Kwon, H. G. Relation between injury of the hypothalamus and subjective excessive daytime sleepiness in patients with mild traumatic brain injury. J Neurol Neurosurg Psychiatry. 87, 1260-1261 (2016).

25. Hanken, K., Eling, P., Kastrup, A., Klein, J. \& Hildebrandt, H. Integrity of hypothalamic fibers and cognitive fatigue in multiple sclerosis. Mult Scler Relat Disord. 4, 39-46 (2015).

26. Alexander, M. P. Mild traumatic brain injury: pathophysiology, natural history, and clinical management. Neurology. 45, 1253-1260 (1995).

27. Solish, N. et al. A comprehensive approach to the recognition, diagnosis, and severity-based treatment of focal hyperhidrosis: recommendations of the Canadian Hyperhidrosis Advisory Committee. Dermatol Surg. 33, 908-923 (2007).

28. Smith, S. M. et al. Advances in functional and structural MR image analysis and implementation as FSL. Neuroimage. 23 Suppl 1, S208-219 (2004).

29. Duvernoy, H. M. \& Bourgouin, P. The human brain : surface, three-dimensional sectional anatomy with MRI, and blood supply. 2nd completely rev. and enl. edn, (Springer, 1999).

30. Jiang, H., van Zijl, P. C., Kim, J., Pearlson, G. D. \& Mori, S. DtiStudio: resource program for diffusion tensor computation and fiber bundle tracking. Comput Methods Programs Biomed. 81, 106-116 (2006).

31. Assaf, Y. \& Pasternak, O. Diffusion tensor imaging (DTI)-based white matter mapping in brain research: a review. J Mol Neurosci. 34, 51-61 (2008).

32. Neil, J. J. Diffusion imaging concepts for clinicians. J Magn Reson Imaging. 27, 1-7 (2008).

33. Godoy, D. A., Panhke, P., Guerrero Suarez, P. D. \& Murillo-Cabezas, F. Paroxysmal sympathetic hyperactivity: An entity to keep in mind. Med Intensiva. 43, 35-43 (2019). 
34. Lee, S. K. et al. Diffusion-tensor MR imaging and fiber tractography: a new method of describing aberrant fiber connections in developmental CNS anomalies. Radiographics. 25, 53-65; discussion 66-58 (2005).

\section{Tables}

Table 1. Demographic data for the patient and control groups

\begin{tabular}{|lll|}
\hline & Patient group & Control group \\
\hline Sex (male:female) & $2: 5$ & $7: 14$ \\
\hline Mean age, years & $51.4(6.7)$ & $48.1(9.8)$ \\
\hline LOC, minutes & $8.1(11.3)$ & - \\
\hline PTA, minutes & $10.6(22.1)$ & - \\
\hline GCS score & $14.9(0.4)$ & - \\
\hline HDSS score & $2.4(0.8)$ & - \\
\hline $\begin{array}{l}\text { Mechanism } \\
\text { of injury }\end{array}$ & Motor vehicle accident & - \\
\hline $\begin{array}{l}\text { Mean duration } \\
\text { to DTI (months) }\end{array}$ & 7 & - \\
\hline
\end{tabular}

Values are mean ( \pm standard deviation), LOC: loss of consciousness, PTA: post-traumatic amnesia, GCS: Glasgow Coma Scale, HDSS: Hyperhidrosis Disease Severity Scale, DTI: diffusion tensor imaging.

Table 2. Severity of hyperhidrosis and presence of clinical features of paroxysmal sympathetic hyperactivity in the individual patients

\begin{tabular}{|lllllll|}
\hline & HDSS & Fever & Tachycardia & Hypertension & Tachypnea & Dystonia \\
Patient 1 & 2 & - & - & - & - & - \\
\hline Patient 2 & 4 & - & - & + & - & - \\
\hline Patient 3 & 3 & - & - & - & + & - \\
\hline Patient 4 & 2 & - & - & + & - & - \\
\hline Patient 5 & 2 & - & - & + & - & - \\
\hline Patient 6 & 2 & - & - & - & - & - \\
\hline Patient 7 & 2 & - & - & - & - & - \\
\hline
\end{tabular}


HDSS: Hyperhidrosis Disease Severity Scale, score 1; sweating is never noticeable and never interferes with daily activities, score 2; sweating is tolerable but sometimes interferes with daily activities, score 3 ; sweating is barely tolerable and frequently interferes with daily activities, and score 4; sweating is intolerable and always interferes with daily activities.

Table 3. Diffusion tensor imaging parameters of the hypothalami in the two study groups

\begin{tabular}{|lllll|}
\hline & \multicolumn{2}{l}{ Fractional anisotropy } & \multicolumn{2}{l|}{ Apparent diffusion coefficient } \\
\cline { 2 - 5 } & Right & Left & Right & Left \\
\hline Patient group & $0.19(0.02)^{*}$ & $0.18(0.03)^{\star}$ & $1.45(0.39)^{\star}$ & $1.53(0.38)^{\star}$ \\
\hline Control group & $0.25(0.03)$ & $0.24(0.03)$ & $1.04(0.15)$ & $1.05(0.16)$ \\
\hline
\end{tabular}

Values represent mean ( \pm standard deviation)

*: $p<0.05$ for comparison between the two groups.

\section{Figures}

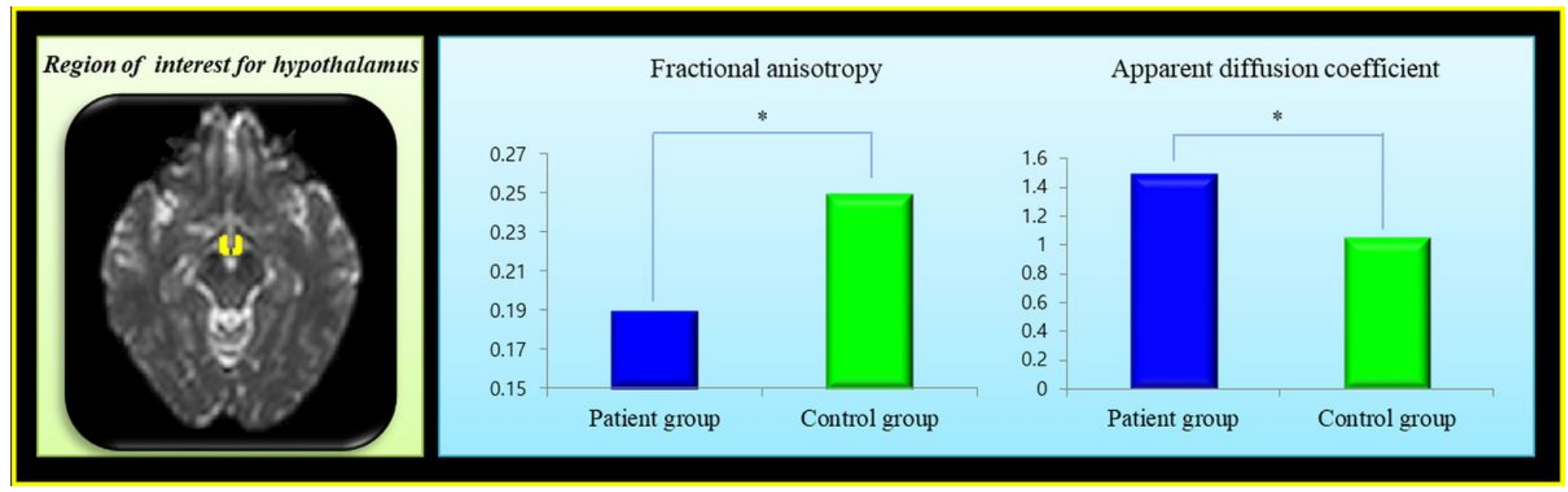

Figure 1

Region of interest for the hypothalamus and results of independent t-test comparison of the fractional anisotropy and apparent diffusion coefficient values between the patient and control groups. $*: p<0.05$ 


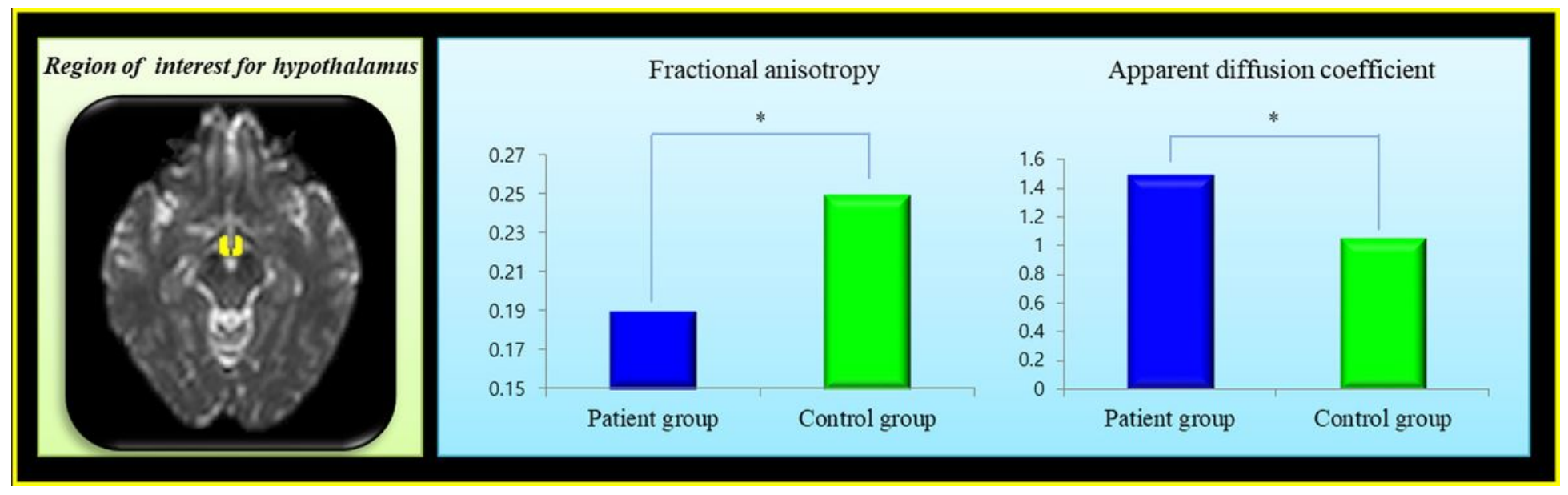

\section{Figure 1}

Region of interest for the hypothalamus and results of independent t-test comparison of the fractional anisotropy and apparent diffusion coefficient values between the patient and control groups. ${ }^{*}: p<0.05$ 